\title{
Doppler ultrasonography combined with transient elastography improves the non-invasive assessment of fibrosis in patients with chronic liver diseases
}

\author{
Tamara Alempijevic ${ }^{1,2}$, Simon Zec ${ }^{1}$, Vladimir Nikolic ${ }^{1}$, Aleksandar Veljkovic ${ }^{3}, Z_{0}$.an Stojanovic ${ }^{2}$, \\ Vera Matovic ${ }^{2}$, Tomica Milosavljevic ${ }^{1,2}$
}

${ }^{1}$ School of Medicine, University of Belgrade, ${ }^{2}$ Clinic for Gastroenterology and Hepatology, Clinical Center of Serbia, ${ }^{3}$ Faculty of Mathematics, University of Belgrade, Belgrad, Serbia

\begin{abstract}
Aims: Accurate clinical assessment of liver fibrosis is essential and the aim of our study was to compare and combine hemodynamic Doppler ultrasonography, liver stiffness by transient elastography, and non-invasive serum biomarkers with the degree of fibrosis confirmed by liver biopsy, and thereby to determine the value of combining non-invasive method in the prediction significant liver fibrosis. Material and methods: We included 102 patients with chronic liver disease of various etiology. Each patient was evaluated using Doppler ultrasonography measurements of the velocity and flow pattern at portal trunk, hepatic and splenic artery, serum fibrosis biomarkers, and transient elastography. These parameters were then input into a multilayer perceptron artificial neural network with two hidden layers, and used to create models for predicting significant fibrosis. Results: According to METAVIR score, clinically significant fibrosis $(\geq F 2)$ was detected in $57.8 \%$ of patients. A model based only on Doppler parameters (hepatic artery diameter, hepatic artery systolic and diastolic velocity, splenic artery systolic velocity and splenic artery Resistance Index), predicted significant liver fibrosis with a sensitivity and specificity of $75.0 \%$ and $60.0 \%$. The addition of unrelated non-invasive tests improved the diagnostic accuracy of Doppler examination. The best model for prediction of significant fibrosis was obtained by combining Doppler parameters, non-invasive markers (APRI, ASPRI, and FIB-4) and transient elastography, with a sensitivity and specificity of $88.9 \%$ and $100 \%$. Conclusion: Doppler parameters alone predict the presence of $\geq F 2$ fibrosis with fair accuracy. Better prediction rates are achieved by combining Doppler variables with non-invasive markers and liver stiffness by transient elastography.
\end{abstract}

Keywords: Doppler ultrasonography, transient elastography, liver fibrosis, METAVIR, artificial neural network

\section{Introduction}

The presence and the severity of liver fibrosis are the main prognostic factors correlated with the risk of developing cirrhosis and its complications in patients with chronic liver diseases [1,2]. Liver biopsy remains the gold standard reference method in assessing the degree of liver fibrosis [3-5]; however, liver biopsy is a costly and

Received 27.07.2016 Accepted 15.09.2016

Med Ultrason

2017, Vol. 19, No 1, 7-15

Corresponding author: Assoc. Prof Tamara Alempijevic, MD, PhD

Clinic for Gastroenterology and Hepatology,

Clinical Center of Serbia, Belgrade

2 Dr Koste Todorovica St., 11000 Belgrade,

Serbia

E-mail: tamara.alempijevic@med.bg.ac.rs invasive procedure with rare, but potentially life-treating complications, including bleeding, pneumothorax, hemothorax, and death [5-7]. In addition, sampling error can occur and extracellular matrix remodelling is a dynamic process susceptible to progression and regression $[8,9]$. Therefore, the use of non-invasive methods for assessing fibrosis has become essential in clinical practice [5]. The best single surrogate marker of liver fibrosis is liver stiffness measurement by transient elastography (TE), which holds an accuracy of over $85 \%$ in diagnosing significant fibrosis (METAVIR F2 or equivalent) [10]. A number of patented and non-patented biochemical tests have shown to be of value for the non-invasive diagnosis of fibrosis. Among the non-patented serum biomarkers, aspartate aminotransferase (AST) to platelet ratio index (APRI), age-spleen-platelet ratio index (ASPRI), and Fibrosis-4 
index (FIB-4) have been shown to be simple and effective in assessing the degree of liver fibrosis $[11,12]$.

Doppler ultrasonography (DUS) is a non-invasive method that has been used for more than 20 years in the evaluation of hepatic blood flow in patients with liver cirrhosis [1]. It can define the velocity and characteristics of blood flow within the portal system and the hepatic and splenic arteries $[2,13]$. Previous studies have shown that portal vein flow velocity $[14,15-18]$ and resistance index of the hepatic [1,14,20-22] and splenic artery [21,23-26] mirror the severity of portal hypertension in patients with cirrhosis. Given these observations, DUS is routinely used in the follow-up of patients with cirrhosis. Nonetheless, few studies have investigated the use of DUS in the assessment of significant liver fibrosis.

We hypothesised that the use of a combination of unrelated methods, namely DUS parameters, liver stiffness by TE and non-patented serum markers of fibrosis, would improve the discriminative ability of TE alone for the non-invasive diagnosis of significant fibrosis $(\geq F$ 2 fibrosis or equivalent). By using liver biopsy as a gold standard reference method, this study was designed to test our hypothesis.

\section{Material and methods}

This study was approved by the Ethical Committee of our hospital in accordance with the Declaration of Helsinki (2000 revision of Edinburgh). We performed an observational retrospective study which included 102 consecutive patients with compensated chronic liver disease, who underwent clinical work-up and liver biopsy for elevated liver function tests (LFTs) from $1^{\text {st }}$ January 2010 to $1^{\text {st }}$ December 2015. All patients were diagnosed and treated at a tertiary care facility. The etiology of disease was based on liver biopsy, and thus patients were classified into one of the following categories: alcoholic, viral [hepatitis B (HBV), hepatitis C (HCV)], autoimmune [autoimmune hepatitis $(\mathrm{AIH})$, primary biliary cholangitis (PBC), primary sclerosing cholangitis (PSC)], metabolic [non-alcoholic fatty liver disease (NAFLD), Wilson's disease, hemochromatosis, $\alpha-1$ antitrypsin deficiency], and cryptogenic.

Patients with acute or acute-on-chronic liver injury: acute alcoholic hepatitis, acute viral hepatitis A, B, or C, drug-induced liver injury, vascular liver disease, alanine aminotransferase (ALT) levels $>5 \mathrm{x}$ upper limit of normal, acute-on-chronic liver failure, decompensated cirrhosis with encephalopathy, variceal bleeding, ascites, or reactivation viral hepatitis were excluded.

All patients received the same work-up consisting of serum biomarkers, DUS examination, TE, and liver biopsy within a time period of 5 days. We collected biochemical parameters: AST, ALT, and platelet count, as well as demographic data for each patient on the first day of admission. Non-invasive scores for the assessment of liver fibrosis (APRI, ASPRI and FIB-4) were calculated using standard formulas at admission (Table I). TE examination was performed in the morning, in patients who had fasted for a minimum of 6 hours, immediately followed by DUS.

\section{Doppler Ultrasonography}

DUS measurements were performed by an experienced hepatologist in the portal trunk, hepatic artery propria and splenic artery using one of either Toshiba Xario SSA-660A or Toshiba Aplio SSA-790 ultrasonographic system (Toshiba, Tokyo, Japan), with a 2-6MHz multifrequency convex probe. Patients who demonstrated the presence of portal vein thrombosis, collateral vessels, suspicion of hepatocellular carcinoma (HCC), or a transjugular intrahepatic portosystemic shunt (TIPS) on ultrasound were excluded.

In the portal venous system, we recorded the diameter and the flow velocity of the portal vein and splenic vein. The diameter, the systolic velocity, the diastolic velocity, and the Resistance Index were measured for the hepatic artery propria and splenic artery. The congestion index was calculated using the formula: $\mathrm{CI}=(\pi \times$ portal vein diameter $\left.{ }^{2} / 4\right)$ /portal vein velocity (PVVel). The resistance index was automatically calculated from the Doppler spectrum as (Vmax - Vmin)/Vmax), where Vmax was the maximum systolic blood flow velocity and Vmin was the maximum end-diastolic velocity. We also measured the splenic craniocaudal diameter.

\section{Transient Elastography}

TE using FibroScan ${ }^{\circledR}$ was performed by an experienced hepatologist using an $\mathrm{M}$ probe, in patients who fasted for at least 6 hours prior to examination, in the supine position, with the right arm in full abduction, on

Table I. Non-invasive indexes and formulas

\begin{tabular}{ll}
\hline Non-invasive index & Formula \\
\hline APRI & $([$ AST/ULN]/platelet count $[109 / \mathrm{L}]) \times 100$ \\
ASPRI & Age $+($ spleen diameter $(\mathrm{cm}) /$ platelets -100$)$ \\
FIB-4 & Age $($ years $) \times$ AST $[\mathrm{U} / \mathrm{L}] /($ platelet count $[109 / \mathrm{L}] \times($ ALT $[\mathrm{U} / \mathrm{L}])$ \\
\hline
\end{tabular}

APRI: aspartate aminotransferase to platelet ratio index, ASPRI: age-spleen-platelet ratio index, FIB-4: Fibrosis-4 index, AST: aspartate aminotransferase, ALT: alanine aminotransferase, ULN: upper limit of normal. 
the mid-axillary line with the probe tip placed in the $9^{\text {th }}$ to $11^{\text {th }}$ intercostal space with a minimum of 10 measurements [27]. Liver stiffness (LS) values were regarded as valid if the following criteria were met: 1) number of valid measurements at least $10 ; 2$ ) a success rate above $60 \%$; and 3 ) an interquartile range (IQR, reflecting the variability of measurements) less than $30 \%$ of the median LS measurements $(\mathrm{M})$ value (IQR/M $\geq 30 \%$ ) [27].

\section{Liver Biopsy}

Biopsy was performed percutaneously under ultrasound guidance using a 17 gauge needle on the mid-axillary line between the $9^{\text {th }}$ and $11^{\text {th }}$ intercostal spaces, with a minimal acceptable specimen length of $15 \mathrm{~mm}$. An experienced specialized liver pathologist evaluated the obtained biopsy specimens. The degree of liver fibrosis was evaluated semi-quantitative according to the METAVIR scoring system and was scored on a scale of $0-4$ as follows: $\mathrm{F} 0=$ no fibrosis, F1 = portal fibrosis without septa, F2 = portal fibrosis and few septa, F3 = numerous septa without cirrhosis, F4 = cirrhosis [28]. Patients were further categorised into two groups according to their METAVIR score; Group 1 included non-significant fibrosis (scores of F0 and F1), and Group 2 included significant fibrosis (F2-4).

\section{Statistical analysis}

By combining Doppler parameters, non-invasive scores and LS values of TE, which were found to have statistically significant correlations to METAVIR score as input values for artificial neural network (ANN), we generated classification models and compared these models in search of a combination of input parameters which would best separate patients with clinically significant and clinically insignificant liver fibrosis. Parameters for the proven quality models were sensitivity, specificity, Area Under the Receiver Operator Curve (AUROC), and absence of data overfitting.

Patients with and without significant liver fibrosis were classified using multilayer perceptron ANN with two hidden layers. The training group consisted of $70 \%$ of the patients, while the remaining $30 \%$ were in the test group, in accordance with the optimal structure of an ANN. The typical structure of a neural network is formed by an input layer, 1 or more hidden layers, and the output layer. The neurons in the input layer receive the data and transfer them to neurons in the first hidden layer through the weighted links. Here, the data are mathematically processed, and the result is transferred to the neurons in the next layer. Ultimately, the neurons in the last layer provide the network's output [29]. For an ANN to function accurately, it needs to be trained, which includes the process by which we add to each training sample input (patient data) and the known output information (correct classification, in our case the presence of significant fibrosis). Af- ter each pair of input-output data passes through the ANN, the computed network output result is compared with the real outcome, and the error rate is then calculated. Based on the error rate, the weight of each connection is adjusted in such a way that the error between the real outcome and the actual output is reduced. An algorithm that makes these adjustments move from layer to layer in a direction from output layer to input layer (the opposite of the usual way data flow through the network) is called a back propagation algorithm and is the most widely used method applied in the process of ANN training [30]. The dataset used for ANN construction and training is randomly partitioned into training and validation samples. The training sample is used to train the network; a validation sample is used to assess the final neural network; the error for this sample gives a good estimate of the predictive ability of the model because the validation cases were not used to build the model, and hence a seemingly small number of patients are used in the validation group [31].

Comparisons between subgroups were made using the Kruskal Wallis test and Pearson correlation. P values less than 0.05 indicated statistical significance. Statistical analysis was performed using SPSS version 22.0.

\section{Results}

Demographic, clinical characteristics, degree of fibrosis by METAVIR, and etiologies of liver disease of patients included in the study are shown in Table II.

\section{DUS parameters}

Mean values of DUS parameters in patients with nosignificant fibrosis (Group 1) and those with significant

Table II. Demographics, degree of fibrosis by METAVIR and etiologies of liver disease.

\begin{tabular}{ll}
\hline & $\mathbf{n}(\mathbf{\%})$ \\
\hline Age (years): mean \pm SD; range & $50.5 \pm 14.6 ; 19-75$ \\
Male & $37(36.3 \%)$ \\
METAVIR & \\
F0 & $27(26.5 \%)$ \\
F1 & $16(15.7 \%)$ \\
F2 & $16(15.7 \%)$ \\
F3 & $13(12.7 \%)$ \\
F4 & $30(29.4 \%)$ \\
Etiology (n=102) & \\
Alcoholic & $8(7.8 \%)$ \\
Autoimmune & $38(37.3 \%)$ \\
Metabolic & $18(17.6 \%)$ \\
Viral & $9(8.8 \%)$ \\
Cryptogenic & $29(28.4 \%)$ \\
\hline
\end{tabular}

SD: standard deviation, n: number of patients, F0: no fibrosis, F1: portal fibrosis without septa, F2: portal fibrosis and few septa, F3: numerous septa without cirrhosis, F4: cirrhosis. 
liver fibrosis (Group 2) are shown in Table III. As shown, most Doppler parameters were similar in the two groups. However, differences were noted in the hepatic artery diameter and hepatic artery systolic velocity, along with the hepatic artery diastolic velocity, splenic artery systolic velocity, and splenic artery resistance index, all of which were higher in the patients with significant fibrosis. The average values of these above mentioned, statistically significant Doppler parameters to the degree of fibrosis by the METAVIR score, are shown in Table IV.

By analysing each individual Doppler parameter, we determined the cut-off values for predicting $\geq \mathrm{F} 2$ fibrosis that are detailed in Table V.
Correlations between Doppler parameters, TE, noninvasive scores and METAVIR score are shown in Table VI. Hepatic artery systolic velocity, hepatic artery diastolic velocity, splenic vein diameter, splenic artery systolic velocity, splenic artery Resistance Index, TE, APRI, and FIB-4 were all found to have statistically significant correlations to the values of METAVIR score.

\section{TE and serum biomarkers}

The optimal liver stiffness cut-off for determining $\geq$ F2 fibrosis by transient elastography was found to be $7.1 \mathrm{kPa}$, with a sensitivity and specificity of $92.0 \%$ and $66.7 \%$, and an AUROC of 0.867. FIB-4 with a cut-off of 1.765 was found to be best at predicting $\geq$ F2 fibro-

Table III. Doppler Ultrasonography means values in patients with and without clinically significant liver fibrosis.

\begin{tabular}{llll}
\hline & Group 1 & P-value \\
\hline HA - diameter (mm) & $4.4 \pm 0.9$ & Group 2 & $\mathbf{0 . 0 3 9}$ \\
HA - systolic velocity (cm/sec) & $84.1 \pm 21.1$ & $4.8 \pm 0.8$ & $\mathbf{0 . 0 0 2}$ \\
HA - diastolic velocity (cm/sec) & $25.3 \pm 8.0$ & $102.8 \pm 30.7$ & $\mathbf{0 . 0 1 4}$ \\
HA - RI & $0.7 \pm 0.06$ & $30.8 \pm 11.7$ & 0.295 \\
PV - diameter (mm) & $12.4 \pm 2.1$ & $0.7 \pm 0.07$ & 0.922 \\
PV - velocity (cm/sec) & $34.1 \pm 9.2$ & $12.5 \pm 2.3$ & 0.155 \\
Spleen - diameter (cm) & $13.2 \pm 3.2$ & $31.4 \pm 8.9$ & 0.088 \\
SV - diameter (mm) & $8.9 \pm 2.2$ & $14.6 \pm 3.6$ & 0.111 \\
SV - velocity (cm/sec) & $35.4 \pm 10.1$ & $9.6 \pm 2.3$ & 0.401 \\
SA - diameter (mm) & $4.9 \pm 1.0$ & $37.4 \pm 10.7$ & 0.483 \\
SA - systolic velocity (cm/sec) & $105.9 \pm 40.9$ & $5.3 \pm 1.4$ & $\mathbf{0 . 0 0 1}$ \\
SA - diastolic velocity (cm/sec) & $42.7 \pm 16.1$ & $132.4 \pm 43.2$ & 0.146 \\
SA - RI & $0.59 \pm 0.06$ & $49.3 \pm 21.4$ & $\mathbf{0 . 0 4 6}$ \\
CI & $0.62 \pm 0.21$ & $0.62 \pm 0.08$ & 0.248 \\
\hline
\end{tabular}

${ }^{a}$ Clinically insignificant fibrosis, ${ }^{b}$ Clinically significant fibrosis, HA: Hepatic Artery, SA: Splenic Artery, PV: Portal Vein, SV: Splenic Vein, SA-RI: Splenic Artery Resistance Index, CI: Congestion Index.

Table IV. Mean values of statistically significant Doppler parameters according to METAVIR score.

\begin{tabular}{|c|c|c|c|c|c|c|}
\hline METAVIR Score & $\begin{array}{l}\text { HA diameter } \\
(\mathrm{mm})\end{array}$ & $\begin{array}{l}\text { HA systolic velocity } \\
\text { (cm/sec) }\end{array}$ & $\begin{array}{l}\text { HA diastolic velocity } \\
(\mathrm{cm} / \mathrm{sec})\end{array}$ & $\begin{array}{l}\text { SA systolic velocity } \\
\text { (cm/sec) }\end{array}$ & SA - RI & CI \\
\hline F0 & 4.3 & 82.8 & 25.7 & 107.9 & 0.59 & 0.64 \\
\hline $\mathrm{F} 1$ & 4.5 & 86.4 & 24.5 & 102.6 & 0.59 & 0.59 \\
\hline $\mathrm{F} 2$ & 4.7 & 94.2 & 26.7 & 126.6 & 0.58 & 0.57 \\
\hline F3 & 4.9 & 101.1 & 32.6 & 127.7 & 0.61 & 0.73 \\
\hline F4 & 4.7 & 108.2 & 32.2 & 137.6 & 0.65 & 0.72 \\
\hline
\end{tabular}

F0: no fibrosis, F1: portal fibrosis without septa, F2: portal fibrosis and few septa, F3: numerous septa without cirrhosis, F4: cirrhosis, HA: Hepatic Artery, SA: Splenic Artery, PV: Portal Vein, SV: Splenic Vein, SA-RI: Splenic Artery Resistance Index, CI: Congestion Index.

Table V. Cut-off values of each individual Doppler parameter

\begin{tabular}{|c|c|c|c|c|}
\hline Doppler parameter & Cut-off & Se $(\%)$ & Sp (\%) & AUROC \\
\hline HA - diameter $(\mathrm{mm})$ & $4.45 \mathrm{~mm}$ & 64.3 & 51.2 & 0.623 \\
\hline HA systolic velocity $(\mathrm{cm} / \mathrm{sec})$ & $98.15 \mathrm{~cm} / \mathrm{s}$ & 53.6 & 78 & 0.658 \\
\hline HA diastolic velocity $(\mathrm{cm} / \mathrm{sec})$ & $28.55 \mathrm{~cm} / \mathrm{s}$ & 51.8 & 68.3 & 0.619 \\
\hline SA systolic velocity $(\mathrm{cm} / \mathrm{sec})$ & $117 \mathrm{~cm} / \mathrm{s}$ & 58.9 & 65.9 & 0.698 \\
\hline SA - RI & 0.615 & 53.6 & 65.9 & 0.609 \\
\hline
\end{tabular}

HA: Hepatic Artery, SA: Splenic Artery, SA-RI: Splenic Artery Resistance Index, Se: Sensitivity, Sp: Specificity, AUROC: Area Under the Receiver Operator Curve. 
Table VI. Correlation between liver stiffness by transient elastography, Doppler parameters, and non-invasive scores with METAVIR.

\begin{tabular}{lll}
\hline & $\begin{array}{l}\text { Pearson } \\
\text { correlation }\end{array}$ & P value \\
\hline HA diameter $(\mathrm{mm})$ & 0.190 & 0.063 \\
HA systolic velocity $(\mathrm{cm} / \mathrm{sec})$ & 0.362 & $<\mathbf{0 . 0 0 1}$ \\
HA diastolic velocity $(\mathrm{cm} / \mathrm{sec})$ & 0.284 & $\mathbf{0 . 0 0 4}$ \\
HA RI & 0.071 & 0.485 \\
PV diameter $(\mathrm{mm})$ & 0.039 & 0.698 \\
PV velocity $(\mathrm{cm} / \mathrm{sec})$ & -0.182 & 0.067 \\
SV diameter $(\mathrm{mm})$ & 0.198 & $\mathbf{0 . 0 5 0}$ \\
SV velocity $(\mathrm{cm} / \mathrm{sec})$ & 0.048 & 0.640 \\
SA diameter $(\mathrm{mm})$ & 0.164 & 0.106 \\
SA systolic velocity $(\mathrm{cm} / \mathrm{sec})$ & 0.293 & $\mathbf{0 . 0 0 3}$ \\
SA diastolic velocity $(\mathrm{cm} / \mathrm{sec})$ & 0.114 & 0.260 \\
SA RI & 0.320 & $\mathbf{0 . 0 0 1}$ \\
CI & 0.183 & 0.066 \\
TE & 0.652 & $<\mathbf{0 . 0 0 1}$ \\
APRI & 0.362 & $<\mathbf{0 . 0 0 1}$ \\
ASPRI & 0.103 & 0.302 \\
FIB-4 & 0.523 & $<\mathbf{0 . 0 0 1}$ \\
\hline
\end{tabular}

HA: Hepatic Artery, SA: Splenic Artery, PV: Portal Vein, SV: Splenic Vein, CI: Congestion Index, RI: Splenic Artery Resistance Index, APRI: aspartate aminotransferase to platelet ratio index, ASPRI: age-spleen-platelet ratio index, FIB-4: Fibrosis-4 index. sis (sensitivity $64.0 \%$ and specificity $86.7 \%$, AUROC 0.775). The APRI cut-off for $\geq$ F2 fibrosis was 0.775 (sensitivity $68.0 \%$ and specificity $86.7 \%$, AUROC 0.780 ) and the cut-off for ASPRI was 2.32 (sensitivity $64.0 \%$ and specificity $66.7 \%$, AUROC 0.581 ).

Average values of age, platelet count, AST and ALT levels depending on the degree of fibrosis as determined by METAVIR score are shown in Table VII.

Combination of unrelated tests: ANNs

Using ANN, a model for the prediction of clinically significant liver fibrosis was made. The distribution of liver fibrosis by METAVIR score between the training and the validation groups is shown in Table VIII, and was not statistically significant, $\mathrm{p}=0.525$. The Hepatic artery diameter, the hepatic artery systolic and diastolic flow velocity, the splenic artery systolic flow velocity and the splenic artery Resistance Index showed the best prediction for fibrosis. Sensitivity of this model alone was of $75.0 \%$ with a specificity of $60.0 \%$. Combining the same Doppler parameters with non-invasive scores for the assessment of liver fibrosis (APRI, ASPRI and FIB-4) gave a much better model for the prediction of clinically significant liver fibrosis as demonstrated by a sensitivity of $81.8 \%$ and a specificity of $85.7 \%$. By adding TE we got a

Table VII. Average values of liver stiffness by transient elastography, age, platelet count, AST and ALT levels according to METAVIR

\begin{tabular}{llllll}
\hline METAVIR Score & Liver Stiffness & Mean age (years) & Mean platelets & Mean AST & Mean ALT \\
\hline F0 & 6.7 & 42 & 203.2 & 34.7 & 60.2 \\
F1 & 10.4 & 48.9 & 201.05 & 62.7 & 88.2 \\
F2 & 10.7 & 46.9 & 194.9 & 67.1 & 98.8 \\
F3 & 51.8 & 179.4 & 83.1 & 102.2 \\
F4 & 15.8 & 52.9 & 131.6 & 69.6 & 70.9 \\
\hline
\end{tabular}

F0: no fibrosis, F1: portal fibrosis without septa, F2: portal fibrosis and few septa, F3: numerous septa without cirrhosis, F4: cirrhosis, AST: aspartate aminotransferase, ALT: alanine aminotransferase.

Table VIII. Distribution of fibrosis by METAVIR score within the training and validation groups of the Artifical Neutral Network.

\begin{tabular}{lllllll}
\hline & \multicolumn{2}{l}{ Metavir Score } & & \multicolumn{2}{c}{ P value } \\
\hline Training group & F0 & F1 & F2 & F3 & F4 \\
Validation group & 19 & 12 & 10 & 11 & 18 & 0.525 \\
& 8 & 4 & 6 & 2 & 12 & \\
\hline
\end{tabular}

F0: no fibrosis, F1: portal fibrosis without septa, F2: portal fibrosis and few septa, F3: numerous septa without cirrhosis, F4: cirrhosis

Table IX. Artificial Neural Network (ANN) Models used for prediction

\begin{tabular}{llll}
\hline & Model used for prediction & & \\
\hline $\begin{array}{l}\text { Prediction of clinically } \\
\text { significant fibrosis }\end{array}$ & Doppler parameters $*$ & $\begin{array}{l}\text { Doppler parameters + APRI, } \\
\text { ASPRI and FIB-4 }\end{array}$ & $\begin{array}{l}\text { Doppler parameters + APRI, } \\
\text { ASPRI, FIB-4 + TE }\end{array}$ \\
\hline Sensitivity & $75.0 \%$ & $81.8 \%$ & $88.9 \%$ \\
Specificity & $60.0 \%$ & $85.7 \%$ & $100.0 \%$ \\
AUROC & 0.827 & 0.948 & 0.990 \\
\hline
\end{tabular}

*Doppler parameters: hepatic artery diameter, hepatic artery systolic velocity, hepatic artery diastolic velocity, splenic artery systolic velocity, splenic arteryresistance index, TE: Transient Elastography, APRI: aspartate aminotransferase to platelet ratio index, ASPRI: age-spleenplatelet ratio index, FIB-4: Fibrosis-4 index, AUROC: Area Under the Receiver Operator Curve 
model that had a sensitivity of $88.9 \%$ and a specificity of $100.0 \%$ (Table IX).

\section{Discussions}

Although liver biopsy remains the gold standard for assessing liver fibrosis, due to its potential for adverse events and contraindications, non-invasive tests are becoming increasingly more important. So far, many noninvasive methods have been developed in order to predict the presence of clinically significant liver fibrosis; however, no single method has been discovered to be superior to the others [32]. In recent years, parameters obtained by DUS of the portal venous system and the hepatic and splenic arteries have been studied in order to find a correlation between these findings and the degree of liver fibrosis.

In this study, we evaluated the possible role of hemodynamic parameters obtained by DUS alone, or added to other unrelated and updated non-invasive tests, in predicting the presence of significant liver fibrosis, using liver biopsy as a gold standard.

The primary result of our study showed that Doppler parameters seem to be of limited value in diagnosing significant fibrosis when used alone, however, their addition to TE and serum markers significantly improved the non-invasive prediction of fibrosis. Therefore, we have developed a model for the prediction of clinically significant liver fibrosis using an ANN approach. With METAVIR scoring by biopsy as a reference standard, Doppler parameters showed to be the best predictors of fibrosis. By employing a combination of non-invasive tests for the assessment of liver fibrosis alongside the Doppler parameters, we obtained a much better model in the evaluation of clinically significant liver fibrosis. The fact that the data in this study was not linearly separable, using ANN as a non-linear statistical data-modelling tool allowed a greater level of precision in classifying patients in with and with no clinically significant fibrosis category. We employed two hidden layers in the structure of ANN, because this was a more precise classification tool when compared to a network with only one hidden layer. Salkić et al used a decision tree, with the parameters classified according to FIB-4 and APRI values, and thus created a similar model for the assessment of liver fibrosis [33].

Majority of our patients were females similar to Glisic et al who also included more female patients in their study [22]. This differs from other countries where the majority of patients were male with viral or alcoholic etiology. This difference in our population sample can be explained by the fact that, in our clinic less patients are treated for viral hepatitis (the majority of our patients are treated by the Infectious Diseases specialist), and the alcoholic liver disease is often diagnosed in later stages. The age range was of 19 to 75 years; cirrhosis (F4) was diagnosed predominantly in older patients, while patients with no liver fibrosis (F0) were generally younger, findings which confirm the results presented by Piscaglia et al [1]. The highest AST and ALT levels were measured in patients with F3 fibrosis, while patients with the lowest values were without any fibrosis (F0). Kelleher et al showed that AST values $>60 \mathrm{IU} / \mathrm{L}$ are strongly associated with significant liver fibrosis [34]. The mean platelet count was the highest for patients with no fibrosis (F0) and the lowest in patients with cirrhosis (F4), which is consistent with the studies conducted by Wai et al [35] and Saitou et al [36].

In our analysis, the average diameter of the HA was the widest in patients with METAVIR stage F3, while the narrowest diameter was seen in patients with F0. Similarly, the lowest average value of HA systolic velocity was demonstrated in patients with F0 while the highest values was seen in patients with cirrhosis. The Systolic velocity in the HA is a good indicator of fibrosis, as confirmed by our data, evidenced by the fact that the average value monotonically increased as the METAVIR score increased from F0 to F4. Patients with F3 fibrosis displayed the highest average value of HA diastolic velocity, whereas the lowest value was seen in patients with F1. Likewise, the highest average value of SA systolic velocity was seen in patients with cirrhosis (F4) and, as expected, the lowest was seen in patients with F1. As concerning the SA-RI average values, the highest was observed in patients with cirrhosis and the lowest in patients at stage F2. The hepatic congestion index was the highest in patients with F3 and F4 and the lowest in patients with F2.

By analysing these results, it was observed that an increased diameter and a higher systolic and diastolic blood flow velocity in the hepatic artery was present in patients with clinically significant liver fibrosis and that the systolic velocity of the hepatic artery increased in patients with liver cirrhosis. This is similar to several studies in the field, which show a progressive increase in the hepatic artery diameter and blood flow in patients with more severe fibrosis, as a result of a progressive arterialisation of the liver circulation $[37,38]$.

The hepatic artery resistance index was shown to have no significant correlation with significant liver fibrosis and cirrhosis in our sample, in contrast to other studies that have shown that HA-RI is in fact, a significant predictor of fibrosis and cirrhosis of the liver [1,19-22,39-42]. This discrepancy may be due to homogeneity in the etiologies of other studies, as compared to the various etiologies in this study; and also due to larger sample size of other in- 
vestigations. Our study however, showed that the systolic velocity and the splenic artery resistance index (SA-RI) was higher in patients with significant fibrosis and cirrhosis. Salvatore et al showed that the SA-RI cut-off of 0.56 enabled the identification of patients with liver stiffness values $>13 \mathrm{kPa}$ obtained by TE (value associated to the presence of cirrhosis) [42], and in two independent studies it was shown that SA-RI was significantly and selectively increased in patients with cirrhosis [25, 43]. Binţinţan et al found that the SA-RI was higher in patients with oesophageal varices than in patients with no varices [44].

TE is by far the most common non-invasive clinically used tool; however, due to its failure in patients with obesity or ascites, it has an applicability of $80 \%$, lower than the serum biomarkers [32]. The biomarkers conversely do not require operator experience and have a higher level of applicability of $>95 \%$ [32]; therefore, our model of combining Doppler parameters and serum biomarkers (APRI, ASPRI and FIB-4), would potentially bridge the gap in problematic patients where TE cannot be performed. Nevertheless, TE and biomarkers are almost equivalent in detecting significant fibrosis, although TE is superior in the detection of cirrhosis [32]. Our triple combination of Doppler, TE and biomarkers, could thereby unify these discordances, particularly inintention-to-diagnose analysis [45].

There were some limitations in our study which should be addressed in future investigations. Firstly, our patient population was heterogenic, with a small number of representative viral hepatitis B or C patients. Therefore, future studies should be etiology specific. In line with this, approximately one third of our patients had biopsy-proven cryptogenic liver disease, which is an astonishingly high number. Global research is currently focused on non-alcoholic liver disease as a frequent cause of cryptogenic cirrhosis [46] and should, therefore, be the focus of future studies. Furthermore, $29.4 \%$ of our patients had liver cirrhosis, which is a large number that has been associated with 'spectrum bias', however, this is not uncommon in a tertiary care facility $[32,47]$. Perhaps the final limitations, are the overall small number of patients included in the analysis and the lack of use of other noninvasive modalities such as $2 \mathrm{D}$-shear wave elastography and 3-D magnetic resonance (MR) elastography, something which can only be improved by conducting larger scale, multi-centric, prospective studies.

\section{Conclusion}

Based on the findings of this study we can conclude that combining DUS parameters with TE and serum markers of fibrosis allows a more accurate prediction of significant liver fibrosis as compared to each single method alone. The model obtained using ANN in which the input components are non-patented serum markers of fibrosis (APRI, ASPRI and FIB-4), liver stiffness measured by TE and Doppler parameters (hepatic artery diameter, systolic and diastolic hepatic artery and systolic splenic artery velocity, and the splenic artery resistance index) had an excellent accuracy for the non-invasive diagnosis of clinically significant liver fibrosis.

Acknowledgements: We would like to thank Professor Annalisa Berzigotti (University Clinic of Visceral Surgery and Medicine, Inselspital, Berne, Switzerland), for her contributions to the final critical review of this manuscript.

Conflicts interest: None to declare.

\section{References}

1. Piscaglia F, Gaiani S, Calderoni D, et al. Influence of liver fibrosis on hepatic artery Doppler resistance index in chronic hepatitis of viral origin. Scand J Gastroenterol 2001;36:647-652.

2. Taylor KJ, Burns PN. Duplex Doppler scanning in the pelvis and abdomen. Ultrasound Med Biol 1985;11:643-658.

3. Nalbantoglu IL, Brunt EM. Role of liver biopsy in nonalcoholic fatty liver disease. World $\mathrm{J}$ Gastroenterol 2014;20:9026-9037.

4. Reiss G, Keeffe EB. Role of liver biopsy in the management of chronic liver disease: selective rather than routine. Rev Gastroenterol Disord 2005;5:195-205.

5. Duarte-Rojo A, Altamirano JT, Feld JJ. Noninvasive markers of fibrosis: key concepts for improving accuracy in daily clinical practice. Ann Hepatol 2012;11:426-439.

6. Rockey DC, Caldwell SH, Goodman ZD, Nelson RC, Smith AD; American Association for the Study of Liver Diseases. Liver biopsy. Hepatology 2009;49:1017-1044.

7. Piccinino F, Sagnelli E, Pasquale G, Giusti G. Complications following percutaneous liver biopsy. A multicentre retrospective study on 68,276 biopsies. J Hepatol 1986;2:165-173.

8. Regev A, Berho M, Jeffers LJ, et al. Sampling error and intraobserver variation in liver biopsy in patients with chronic HCV infection. Am J Gastroenterol 2002;97:2614-2618.

9. Siddique I, El-Naga HA, Madda JP, Memon A, Hasan F. Sampling variability on percutaneous liver biopsy in patients with chronic hepatitis C virus infection. Scand J Gastroenterol 2003;38:427-432.

10. Friedrich-Rust M, Ong MF, Martens S, et al. Performance of transient elastography for the staging of liver fibrosis: a meta-analysis. Gastroenterology 2008;134:960-974.

11. Abdollahi M, Pouri A, Ghojazadeh M, Estakhri R, Somi M. Non-invasive serum fibrosis markers: A study in chronic hepatitis. Bioimpacts 2015;5:17-23. 
12. Khairy M, Abdel-Rahman M, El-Raziky M, et al. Non-invasive prediction of hepatic fibrosis in patients with chronic $\mathrm{HCV}$ based on the routine pre-treatment workup. Hepat Mon 2012;12:e6718.

13. 1Taylor KJ, Burns PN, Woodcock JP, Wells PN. Blood flow in deep abdominal and pelvic vessels: ultrasonic pulsedDoppler analysis. Radiology 1985;154:487-493.

14. Iranpour P, Lall C, Houshyar R, et al. Altered Doppler flow patterns in cirrhosis patients: an overview. Ultrasonography 2016;35:3-12.

15. Ohnishi K, Saito M, Nakayama T, et al. Portal venous hemodynamics in chronic liver disease: effects of posture change and exercise. Radiology. 1985;155:757-761.

16. Zironi G, Gaiani S, Fenyves D, Rigamonti A, Bolondi L, Barbara L. Value of measurement of mean portal flow velocity by Doppler flowmetry in the diagnosis of portal hypertension. J Hepatol 1992;16:298-303.

17. Cioni G, D'Alimonte P, Cristani A, et al. Duplex-Doppler assessment of cirrhosis in patients with chronic compensated liver disease. J Gastroenterol Hepatol 1992;7:382384.

18. Gaiani S, Bolondi L, Li Bassi S, Zironi G, Siringo S, Barbara L. Prevalence of spontaneous hepatofugal portal flow in liver cirrhosis. Clinical and endoscopic correlation in 228 patients. Gastroenterology 1991;100:160-167.

19. Colli A, Cocciolo M, Mumoli N, Cattalini N, Fraquelli M, Conte D. Hepatic artery resistance in alcoholic liver disease. Hepatology 1998;28:1182-1186.

20. Sacerdoti D, Merkel C, Bolognesi M, Amodio P, Angeli P, Gatta A. Hepatic arterial resistance in cirrhosis with and without portal vein thrombosis: relationships with portal hemodynamics. Gastroenterology 1995;108:1152-1158.

21. Piscaglia F, Zironi G, Gaiani S, et al. Systemic and splanchnic hemodynamic changes after liver transplantation for cirrhosis: a long-term prospective study. Hepatology 1999;30:58-64.

22. Glisic TM, Perisic MD, Dimitrijevic S, Jurisic V. Doppler assessment of splanchnic arterial flow in patients with liver cirrhosis: correlation with ammonia plasma levels and MELD score. J Clin Ultrasound 2014;42:264-269.

23. Bolognesi M, Sacerdoti D, Merkel C, et al. Splenic Doppler impedance indices: influence of different portal hemodynamic conditions. Hepatology 1996;23:1035-1040.

24. Bolognesi M, Sacerdoti D, Bombonato G, et al. Splenic impedance indices: a useful method to monitor patients after liver transplantation? Hepatology 1998;27:674-678.

25. Piscaglia F, Donati G, Cecilioni L, et al. Influence of the spleen on portal haemodynamics: a non-invasive study with Doppler ultrasound in chronic liver disease and haematological disorders. Scand J Gastroenterol 2002;37:12201227.

26. Perisic MD, Culafic DjM, Kerkez M. Specificity of splenic blood flow in liver cirrhosis. Rom J Intern Med 2005;43:141-151.

27. Castera L, Forns X, Alberti A. Non-invasive evaluation of liver fibrosis usingtransient elastography. J Hepatol 2008;48:835-847.
28. Bedossa P, Poynard T. An algorithm for the grading of activity in chronic hepatitis C. The METAVIR Cooperative Study Group. Hepatology 1996;24:289-293.

29. Amato F, López A, Peña-Méndez EM, Vaňhara P, Hampl A, Havel J. Artificial neural networks in medical diagnosis. J Appl Biomed 2013;11:47-58.

30. Rojas RL. The back propagation algorithm. Neural networks: a systematic introduction. Berlin (Germany)/New York (NY): Springer-Verlag; 1996:151.

31. Jovanovic P, Salkic NN, Zerem E. Artificial neural network predicts the need for therapeutic ERCP in patients with suspected choledocholithiasis. Gastrointest Endosc 2014;80:260-268.

32. European Association for Study of Liver; Asociacion Latinoamericana para el Estudio del Higado. EASL-ALEH Clinical Practice Guidelines: Non-invasive tests for evaluation of liver disease severity and prognosis. J Hepatol 2015;63:237-264.

33. Salkic NN, Cickusic E, Jovanovic P, et al. Online combination algorithm for non-invasive assessment of chronic hepatitis B related liver fibrosis and cirrhosis in resourcelimited settings. Eur J Intern Med 2015;26:628-634.

34. Kelleher TB, Mehta SH, Bhaskar R, et al. Prediction of hepatic fibrosis in $\mathrm{HIV} / \mathrm{HCV}$ co-infected patients using serum fibrosis markers: the SHASTA index. J Hepatol 2005;43:78-84.

35. Wai CT, Cheng CL, Wee A, et al. Non-invasive models for predicting histology in patients with chronic hepatitis B. Liver Int 2006;26:666-672.

36. Saitou Y, Shiraki K, Yamanaka Y, et al. Noninvasive estimation of liver fibrosis and response to interferon therapy by a serum fibrogenesis marker, YKL-40, in patients with $\mathrm{HCV}$-associated liver disease. World J Gastroenterol 2005;11:476-481.

37. Yamauchi H, Suda Y, Yamamoto K, Sato T. Arteriographic studies of splenic and hepatic arteries in portal hypertension. Tohoku J Exp Med 1970;101:363-374.

38. Toni R, Bolondi L, Gaiani S, et al. Accessory ultrasonographic findings in chronic liver disease: diameter of splenic and hepatic arteries, fasting gallbladder volume, and course of left portal vein. J Clin Ultrasound 1985;13:611-618.

39. Abhilash H, Mukunda M, Sunil P, Devadas K, Vinayakumar KR. Hepatic artery duplex Doppler ultrasound in severe alcoholic hepatitis and correlation with Maddrey's discriminant function. Ann Gastroenterol 2015;28:271-275.

40. Tanaka K, Numata K, Morimoto M, et al. Elevated resistive index in the hepatic artery as a predictor of fulminant hepatic failure in patients with acute viral hepatitis: a prospective study using Doppler ultrasound. Dig Dis Sci 2004;49:833842.

41. Numata K, Tanaka K, Kiba T, et al. Hepatic arterial resistance after mixed-meal ingestion in healthy subjects and patients with chronic liver disease. J Clin Ultrasound. 1999;27:239-248.

42. Salvatore V, Borghi A, Peri E, et al. Relationship between hepatic haemodynamics assessed by Doppler ultrasound and liver stiffness. Dig Liver Dis 2012;44:154-159. 
43. Tarantino G, Spano A, Loi G, et al. Is spleen circulation impaired in systemic sclerosis and what is the role of liver fibrosis? World J Gastroenterol 2011;17:1606-1613.

44. Bintintan A, Chira RI, Bintintan VV, et al. Value of hepatic elastography and Doppler indexes for predictions of esophageal varices in liver cirrhosis. Med Ultrason 2015;17:5-11.

45. Zarski JP, Sturm N, Guechot J, et al; ANRS HCEP 23 Fibrostar Group. Comparison of nine blood tests and transient elastography for liver fibrosis in chronic hepatitis $\mathrm{C}$ : The ANRS HCEP-23 study. J Hepatol 2012;56:55-62.

46. Pais R, Barritt AS Th, Calmus Y, et al. NAFLD and liver transplantation: Current burden and expected challenges. J Hepatol 2016 Jul 30; doi: 10.1016/j.jhep.2016.07.033.

47. Degos F, Perez P, Roche B, et al. Diagnostic accuracy of FibroScan and comparison to liver fibrosis biomarkers in chronic viral hepatitis: a multicenter prospective study (the FIBROSTIC study). J Hepatol 2010;53:1013-1021. 